\title{
THE INTRACAPSULAR EXTRACTION OF CATARACT
} An Analysis of 67 Cases as a plea for its wider adoption

BY

R. C. J. MEyer, M.D., F.R.C.S.Ed.

OPHTHALMIC SURGEON, JOHANNESBURG HOSPITAL, CLINICAL LECTURER, WITWATERSRAND UNIVERSITY, SOUTH AFKICA

THE intracapsular extraction of cataract has long appealed to many ophthalmic surgeons. Formerly it was considered necessary to wait until a cataract became mature, and the patient was often condemned to an indeterminate period of reduced amenity and crippled activity due to impaired vision. Hence it is that the operation of extracting the cataract, mature or immature, in its capsule makes a powerful appeal to many ophthalmic surgeons.

The purpose of this paper is to put before the reader the results of a series of attempts on the part of the writer, and to leave him to judge for himself whether or not the operation described is worth taking up. The operation is substantially that which has been done in Europe and America for many years.

My interest was originally aroused by a film shown by Dr. Sinclair, of Edinburgh, at the Ophthalmological Congress in London in 1925. In the same year I saw an intracapsular extraction performed at Edinburgh by Dr. Traquair. Subsequently the results published by Professor Elschnig, of Prague, proved a powerful stimulus. To him and to Dr. Traquair I wish to express my intense gratitude for explaining the operation to me in personal letters, and for help in connection with the literature.

The essence of this operation is that the surgeon takes over complete control of the eye from the patient. This is not achieved by the will power of the patient, but only by the correct use of novocaine injections, and stitches to control the muscles likely to offend.

\section{Technique of the Operation}

The usual attention is paid to the health of the patient, and to the eye, and the same preparation for operation as for extracapsular extraction is carried out. Two hours before operation a sedative is given, e.g., chloral and potassium bromide, $12 \frac{1}{2}$ grs. of each. (Opium and its derivatives have too often been disappointing, and make the patient sick). The patient enters the theatre less nervous and less agitated, and his subsequent behaviour on the table is more lethargic, hence there are no sudden movements to embarrass the surgeon. One hour before operation the pupil is dilated by homatropine and cocaine drops. 
Fifteen minutes before reaching the operating table, the process of rendering the eye anaesthetic begins:-

Cocaine hydrochl. 2 per cent. solution 2 minims.

Cocaine hydrochl. 3 per cent. solution 2 minims two minutes later.

Adrenaline 1 in 1,000 solution 2 minims two minutes later.

These two latter are used alternatively every two minutes, i.e., the eye has had cocaine at least five times and adrenaline at least three times before being brought to the theatre. Here the patient gets a few more drops of each, and two drops of cocaine into the fellow eye. Tincture of iodine or similar antiseptic is applied to the skin of the face, to as much of the field as is not subsequently covered by towels or gauze.

1. Paresis of Orbicularis Palpebrarum.-Novocaine 1 per cent. with adrenaline solution $1 / 10,000$ is now injected to paralyse the orbicularis palpebrarum muscle, incidentally also anaesthetizing that region. Twenty minims of the solution are ample. The needle is inserted about 1 centimetre external to the external canthus, and slightly below it, some fluid is injected slightly upwards to catch the nerve supply to the upper fibres of the orbicularis, a little more slightly downwards to catch the lower fibres, and the largest quantity with the needle directed backwards and downwards towards the lobule of the ear, but inserted more deeply, to catch the diverging fan of fibres of the facial nerve.

1a. To ensure Post-operative Closure of Eyelids.-After withdrawing the needle, two minims of the same solution may be injected just above the cilia, opposite the middle of the margin of the upper lid, and a stitch inserted, chiefly to use for complete closure of the lids after the operation, before the paralysing effect of the novocaine on the orbicularis has disappeared. This suture is also useful to lift up the lid during the course of the operation, and in the post-operative inspections. The conjunctival sac is irrigated with a mild mercurial antiseptic, followed by normal saline.

2. Control of Eyeball.-A stitch is now to be inserted below or into the tendon of the superior rectus. This stitch and suture give the surgeon wonderful control of the eyeball. Five minims of the same 1 per cent. novocaine, and $1 / 10,000$ adrenaline in solution may be injected subconjunctivally into the region of the insertion of the muscle if the surgeon is not satisfied with the cocaine anaesthesia already present.

3. Complete Anaesthesia of Eyeball and Partial Anaemia of Uvea.-The retrobulbar injection of $8 \mathrm{minims}$ of 2 per cent. novocaine, and $1 / 1,000$ suprarenin is now made by inserting the hypodermic needle deeply into the orbit just above its lower outer angle, through or almost below the lower lid, aiming for a point as near the entrance of the optic nerve into the eyeball as is possible without touching the optic nerve itself. It is easy to verify the 
freedom of the eyeball and the optic nerve from any part of the needle by asking the patient to move the eye in various directions before gently injecting the fluid; indeed, slow movement of the eye during the injection ensures the novocaine reaching most of the ciliary nerves and vessels surrounding the optic nerve. It is important to limit the amount of fluid injected; the lack of that knowledge caused four eyes to be lost through vomiting followed by expulsive haemorrhage.

The lid speculum is now inserted. It is important to use a variety that lifts itself off the eyeball even if it should be a little more clumsy in appearance and in insertion and removal, and even if it does have parts which sometimes appear to be slightly in the way of the surgeon. The speculum of Olah is used by us, and though it has some of the disadvantages just indicated, its ability to lift itself off the eyeball and the absolute impossibility of any spasm of the orbicularis when it is in use is more than adequate compensation for its faults. Dr. Traquair has devised a lighter speculum without the above disadvantages.

4. Conjunctival Flap.- The incision may now be made, but owing to the importance of obtaining a good conjunctival flap, I have lately taken to preparing the latter before using the Graefe knife. Any sized conjunctival flap can be prepared with scissors beforehand, but it is not advisable to make it too big, as it rather hinders the view of the knife in the anterior chamber unless the flap is skilfully held out of the way by the assistant. If too big a flap is laid back flat on the sclera before the limbal incision is made, I found that the knife in making the counterpuncture generally made a second smaller conjunctival flap, thus defeating the object of preparing the flap beforehand.

5. Incision.-The ordinary limbal Graefe incision is now made, but bigger than usual. Three-fifths or three-quarters of $180^{\circ}$ hardly seems sufficient; the lens in its capsule is so much bigger than the ordinary nucleus that one is almost startled by its magnitude. The capsule is likely to tear in bringing the lens out of too small an incision and though the ordinary incision can easily be enlarged by a sharp Graefe knife cutting each end downwards, or by the more clumsy scissors, it is certainly more satisfactory to make the incision large enough at the commencement. Only eyes with very shallow anterior chambers require the outward enlargement of the incision, and in some rare cases even the entire making of it from the outside. It is too late to enlarge the incision during the delivery of the lens as both hands are then fully occupied, and it is a pity to tear the capsule just at the last moment.

I have never found it necessary to make an incision of fully $180^{\circ}$ but have had good cause to regret having made it $135^{\circ}$. On the whole $150^{\circ}$ to $160^{\circ}$ should be sufficient. The healing process in the 
cornea does not seem to be retarded by the size of the incision. The less scleral the incision is, the less is the haemorrhage. Should any blood get in, it is advisable to irrigate it out before it has had time to clot and adhere to the iris, and it does not take thirty seconds to stick to the iris quite tenaciously. Having removed all blood from the anterior chamber and having stopped any source of haemorrhage by applying adrenaline pledgets, we are free to proceed with the iridectomy. There are three varieties.

6. Iridectomy. - Professor Elschnig uses a small transverse incision into the junction of iris and ciliary body, and is satisfied with its results. Others use the peripheral iridectomy to which we are accustomed in the Hess extraction out of the capsule. Then there is the complete iridectomy.

Personally, I have never had the courage to rely on Elschnig's "iris-root" incision, but have been very pleased with the peripheral iridectomy which appeals much more to me than the complete iridectomy, as the latter by cutting the sphincter pupillae spoils not only the cosmetic result, but, far worse, does not give the sphincter the chance to pull the iris away so well from the limbal incision. A peripheral iridectomy $2.3 \mathrm{~mm}$. broad, but hardly as long, seems to achieve its object well enough. It is true that one sometimes has to replace a prolapsed iris, or even abscise it, but the proportion is small.

7. Conjunctival. Sutures.-The next step would seem to be the extraction of the lens, but this should be postponed till after the conjunctival stitches have been inserted, generally two, one each at the junction of the middle third, with the outer and inner thirds, or slightly farther out if the surgeon prefers to insert others after the extraction of the lens. It is good practice to include in the stitches a good piece of the conjunctiva, both limbal and peripheral, so that if need be, these sutures can be used to exert a certain amount of traction to bring the two lips of the limbal wound together fairly firmly. A preliminary loose knot is made on each side but not tied, and the portion of the suture overlying the incision is pushed out of the way by making a rather long loop. There is no doubt that these sutures do get in the way while extracting the lens entire, unless they are made long and kept out of the way of the incision.

8. The Lens Extraction. - It is well to pause a moment to see that everything is ready, to clear the suture loops out of the way, to run a thin iris repositor along the whole incision to make sure there are no uncut subconjunctival fibres which might hinder the free exit of the lens, to see that there is no agglutination of the edges of the wound by fibrin from the unavoidable though possibly very small amount of haemorrhage, and to make sure that the view of the lens surface is unembarrassed by blood or iris pigment. Irrigation of anterior chamber with normal saline ensures a clear picture, if such is not previously present. 
The assistant is ready with thin straight forceps in each hand to push or lift any embarrassing structure out of the way. The surgeon takes a tenotomy hook, or variation of it, in his left hand, and the capsule forceps in the right. Of capsule forceps for grasping the lens capsule without rupturing it, there are several varieties, the main requirement of each being a smooth tip, neither cutting nor tearing, yet holding on. The next requirement is the correct angle to enable the reversal of the lens surfaces to be achieved by the forceps.

As in all senile cataract extractions, here too the surgeon takes the precaution of having various spoons and loops. ready in case there are vitreous complications.

The forceps slides down behind the cornea into the pupil and behind its lower margin, so as to lie between iris and lens capsule but not beyond the latter. In the last two or three millimetres of its course, it is slid down with the points open, but not beyond a maximum of three millimetres, two are better. The points are then depressed gently about one millimetre into the substance of the lens and gently closed grasping the capsule. It is better to be too deliberate or too gentle than the reverse. Coarse handling ruptures the capsule and the operation becomes the ordinary extracapsular extraction; gentle handling can be repeated until the capsule is grasped successfully. This can be both felt and also seen by the light reflexes from the folded capsule grasped in the forceps. Now the suspensory fibres of the lens have to be ruptured without tearing the capsule. This is achieved by gentle swaying or displacement of the forceps holding the lens, towards each side for $1-2 \mathrm{~mm}$. It is advisable to take about twenty seconds over this process. When a fair number of lateral suspensory fibres have thus been ruptured, and this can only be surmised by watching the movement that one has oneself imparted to the lens, the lower ones are then ruptured by gentle stretching. The extraction of the lens in its capsule now really commences. The capsule forceps having once gripped the capsule never lets go and now begins to rotate the lower extremity of the lens forwards so that ultimately this comes out of the incision first. In other words, the lens is rotated almost $180^{\circ}$ around a horizontal axis parallel to a line joining the external and internal canthi and comes out with its posterior surface lying against the cornea. This process of rotation automatically ruptures the remaining superior and inferior suspensory fibres of the lens by overstretching them. The limiting membrane of the vitreous, perhaps not demonstrable microscopically, but clinically a very decided entity, tolerates this procedure well and neither it nor the lens capsule break nor tear if the manipulations are gentle. But the capsule, that very small bit of it that is grasped, cannot be expected to hold and pull the whole of the lens out without any 
external assistance. The moment, therefore, that the forceps begins rotating the lens from below, we start pushing the lens from behind and helping it with the tenotomy hook (or its variation), pressing first in the region of the limbus below and, as we see and feel the lens coming, the instrument gradually follows up and pushes along the lens by gentle pressure exerted on the limbus and cornea at the part where the edge of the lens is lying at each instant in its course behind the cornea. This direction of application of this pressure is, of course, such that together with the lesser traction of the capsule forceps the lens is pushed towards the incision. Thus even in the extraction in the capsule we also resort to expression, which latter should be the correct name for the old operation of extraction.

Gradually the wound opens wider and wider, perhaps slightly encouraged by the assistant holding or pushing obstructions such as the conjunctival flap out of the way, and we see the glistening smooth surface of the lens capsule presenting. Once we realize that the major diameter of the lens is passing through the wound the pressure behind is lessened, and, once it has passed, gentle rotation or a swaying movement to and fro of the lens disengages it from the lips of the wound and it is removed entire. By now the pressure on the eyeball has long been stopped and the two conjunctival sutures are immediately pulled tight and tied in order to prevent any risk of loss of vitreous. The surgeon may now attend to the toilet of the wound.

9. Toilet of the Wound.-The iris is replaced by pushing the repositor under the flap and further sutures are put in if it is thought necessary. It is advisable to consider the utility of any suture as a tension stitch, if there does not seem perfect co-aptation of the wound.

When the pupil is quite round again (some gentle persuading of the iris by the repositor may be required before this is achieved) the superior rectus stitch is withdrawn and the speculum removed, the conjunctival sac is rinsed out with saline, and two drops of eserine solution instilled. One may encourage the patient by showing him that the eye can now count fingers and after that he is asked "gently to close both eyes." He cannot completely close the operated eye on account of the paresis of the orbicularis palpebrarum, but the stitch in the upper lid is now fixed to the cheek with adhesive plaster and keeps the two lids well apposed. The bandage is put on and the patient wheeled back to bed. The bandage may be uniocular or binocular. I have generally used the binocular Moorfields bandage for the first day as the patient then does not attempt to move the unoperated and with it the operated eye.

10. Post-operative Treatment.-The post-operative treatment is 
the same as that of an extracapsular extraction, except that it is probably wiser to use eserine at the first dressing next morning. The pupil dilates equally well after the use of eserine for two days. Atropine can then be instilled. I have had no bad effects from the patient sitting up in an easy chair next day, and this treatment shortens the usual complaints due to the discomfort of being afraid to strain when in bed. In fact it is the routine practice unless any complications have set in during the first twenty-four hours.

I can confidently recommend the conjunctival suture as a great source of relief from anxiety to the surgeon as regards post-operative sequelae, in fact all his troubles occur while the patient is on the operating table, where he has every advantage in his favour, and the eye is practically immobile and totally anaesthetized. The stitches either come out of their own accord from the sixth to the fourteenth day, according to the depth of their insertion, or may be removed.

\section{Results of 67 Cases}

The analysis of the cases is very interesting, reflecting as it does, the psychological effect of failure and success.

My losses were heavy, too heavy to continue the operation if the fault could not be remedied. But of the five cases I lost, the fault could easily be adjusted now in the light of the experience gained, and the reader, by avoiding my mistakes, need have none of these losses. I lost the fourth case through not possessing a self-lifting speculum ; at the critical moment when the vitreous presented after delivery of the lens, my inexperienced assistant unfortunately depressed the speculum instead of elevating it, and the vitreous shot out.

I did three extracapsular extractions after this failure before I could summon up courage to make the fifth intracapsular attempt ; in this the capsule burst early, so that it became an easy extracapsular extraction. At the sixth attempt the capsule ruptured on delivery of the lens (the incision had been too small), still it was a step onwards ; the seventh was successful, but in the eighth I again ruptured the capsule on delivery of the lens.

In the ninth to thirteenth attempts I burst the capsule early; they all became easy extracapsilar extractions.

One was obviously losing heart, for by now, in addition to the above, seven extracapsular extractions had also been done by the ordinary method.

The fourteenth attempt achieved a complete intracapsular extraction and from the sixteenth the results improved. I lost vitreous in the eighteenth case, but a subsequent iridotomy gave $6 / 9$ vision. 
Everything went satisfactorily until I lost vitreous in the twentyninth case ; the patient began to vomit two hours after the operation, producing expulsive haemorrhage. I lost that eye. I blamed the loss of vitreous and his blood pressure, 185/100, but the next three losses taught me otherwise.

I lost the forty-second, forty-sixth and forty-seventh cases by expulsive haemorrhage; 42 and 46 lost vitreous on the table, 47 did not and had a blood pressure of only 120/85. All four counted fingers on the table but began to vomit a couple of hours after the operation and continued to do so until expulsive haemorrhage had destroyed the eye. This was too much! It occurred to me that I might be using too large a retrobulbar injection. I therefore limited the amount to a maximum of 8 minims of 2 per cent. novocaine and in the last 21 cases there have been no complaints of sickness, still less of vomiting. Thus the five losses (i.e., 7.5 per cent.) cannot be set down as a fault of the operation but rather to the lack of skill and knowledge of the surgeon. I am happy to say they all had a remaining good eye either previously or later successfully deprived of its cataract.

Now having explained the losses, let me classify the remaining 62 cases.

34 were removed in the capsule- 51 per cent.

8 burst the capsule at the moment of delivery -12 per cent.

20 burst the capsule early, i.e., became ordinary extracapsular extractions-30 per cent.

The late bursting of the capsule can almost be counted as a complete removal since the lower two-thirds of the pupil usually remain jet black and the little remaining cortex if not easily removable at the time by spoon, irrigation or suction syringe, does not embarrass vision, though it does delay the healing of the eye and causes much more redness than the complete removal of the lens in its capsule.

One has reasonable hopes of reducing the figure of 30 per cent., although, when it is remembered that these figures embrace all kinds of cataracts, and that a young capsule (under 40 years) and also a very tight one (with swollen cortex) rupture more easily, it would be easy with selected cases to get a much higher percentage of complete intracapsular extractions.

\section{Other Complications}

Prolapsed Irides. - There were five, none large. One was massaged back into position with the help of eserine, and two were replaced with the repositor, the patients remaining in bed, and two on the operating table.

Iridotomies.-Four had to be performed, the pupil having been drawn up by escaping vitreous in two cases, the third where in 
spite of a complete iridectomy on the table, the iris subsequently became drawn up, and the fourth where fear of damaging the presenting vitreous at the operation prevented me from getting the pupil back to normal shape. Ther all got 6/5-6/12 vision.

Hyphaemata. - These seemed, if anything, fewer but more severe than with the extracapsular extraction. When they did occur they were always traced to a definite trauma during the first six days of convalescence.

In three cases the anterior chamber filled completely with blood so much so that there was not even perception of light after the accident. The stitches allowed the conjunctival flap to bulge but they never gave way and evidently stopped the haemorrhage. Naturally one was very anxious about the blood getting into the vitreous but this did not occur, the blood was all absorbed fairly fast and left no after effects. They all had undilated pupils and 6/6 vision six weeks after the operation. Apparently the limiting membrane of the vitreous does not rupture with the gradual but uniform increase of pressure.

High Tension.-One patient developed high tension some months after the operation but this yielded very well to a cyclodialysis.

One case of successful extraction in the capsule was a swollen cataractous lens producing acute glaucoma in a woman of 58 years. The operation was difficult but the patient has now got a round pupil and $6 / 9$ vision.

Loss of Vitreous. - In my first attempt at intracapsular extraction I lost vitreous. Owing to over-gentle holding of the unruptured capsule with the forceps I let the capsule go. I was too timid to get a grip on the apparently tense and very shining capsule of the lens, of which by now fully one-third was delivered. I had to rely on expression and this was too forcible, and I expressed some vitreous with the intact lens. The result was $6 / 12$ vision with a plus $8.0 \mathrm{D}$. sphere and plus $6.0 \mathrm{D}$. cylinder three months later. At my fourth attempt I lost vitreous by the inopportune depressing of the speculum, and with it I lost the eye as before mentioned among the complete losses.

The twenty-ninth and forty-sixth cases lost vitreous on the table, probably by clumsiness on my part. They could count fingers on the table after the operation but began vomiting two hours after the operation, and I lost these two eyes and the forty-seventh by expulsive haemorrhage. Had the forty-seventh case also lost vitreous, which it did not, and had it taken more than half-an-hour to do, I might have regarded the expulsive haemorrhages as due to that cause; fortunately for my subsequent cases, the true cause, too large a retrobulbar injection, came to light over this very case.

Presenting of Vitreous without Rupturing.-This is a very unpleasant complication. It occurred twice, and the anxiety lest the 
external limiting membrane of the vitreous should give way during the inserting of the stitches to pull the gaping sclerocorneal wound together is exhausting. In neither case did it give way, yet one cannot hope for such good fortune in every case. The latter two were post-operative complications and have had the direct result of making the conjunctival stitch at the operation more of the nature of a tellsion stitch.

Of Other or Delayed Bad Results.-I have seen none beyond those above mentioned, and my first attempt was made in November, 1928.

The ADVANTAGES of the OPERATION are:-

1. Applicability to immature cataract, which is technically easier than swollen mature cataract.

2. Absolute lack of irritation of eye during convalescence. This is too striking for words; it must be due to there being absolutely no capsule or cortex to irritate the eye, which remains quiet and white, showing no ciliary irritation whatsoever.

3. Early rupture of the lens capsule merely reduces the operation to the old and well-known extracapsular extraction. The capsule ruptures either early or on delivery, in either case it does not matter. I have never had one rupture half way, and this is one's main fear; apparently it is groundless.

Disadvantages:-

1. Technically it is not so easy as the extraction out of the capsule. It is not a beginner's operation, but well within the reach of any surgeon of average capability.

2. The duration of operation is longer owing to the novocaine injecting and sewing of the conjunctiva, though the sedative to the patient, and the novocaine apparently keep him quiet, and the eye almost immobile.

3. There is no capsule to hold back the vitreous.

The older operations of intracapsular extraction have failed to give satisfactory results in the hands of others than their originators. Here is an operation which has stood the test at the hands of an operator of only moderate ability, who had to learn it chiefly from two or three letters from its exponents.

There must be an enormous number whose opportunities and experience are so much greater than those of the writer, that they can have no hesitation in trying and adopting this operation, which promises earlier and easier relief, and may be considered a distinct step in the advance of ophthalmology.

If the analysis of these results should result in a wider adoption of this operation, it will have achieved a useful purpose.

REFERENCE

Kubik.-Klin. Monatsbl. f. Augenheilk., May, 1929. 